\title{
Effects of supplementation with polyunsaturated fatty acids in patients with heart failure
}

\author{
Savina Nodari $\cdot$ Marco Triggiani $\cdot$ Alessandra Manerba $\cdot$ \\ Giuseppe Milesi • Livio Dei Cas
}

(C) SIMI 2011

\begin{abstract}
Despite the clinical and prognostic improvement obtained with the current medical treatment, heart failure (HF) continues to have high morbidity and mortality and its prevalence is increasing in most regions of the world. Thus, there is a need for novel adjunctive therapies that act independently of current neurohormonally and haemodynamically oriented drugs. Nutritional approaches are particularly attractive because they could work additively with established therapies without negative hemodynamic effects. There is growing evidence that omega- 3 polyunsaturated fatty acids (n-3 PUFAs) supplementation positively impacts established pathophysiological mechanisms in HF and thus has a potential role for preventing and treating HF. The results of the GISSI-HF trial have indicated that, in patients with chronic HF on evidence-based therapy, long term treatment with PUFAs reduced mortality and hospitalizations for cardiovascular reasons, irrespective of etiology and left ventricular (LV) ejection fraction (EF). The purpose of this review is to summarize the evidence emerged from studies conducted so far on the effect of $n-3$ PUFAs in HF.
\end{abstract}

Keywords n-3 PUFAs · Heart failure - Metabolic therapy · Left ventricular function · Non-ischemic cardiomyopathy

\section{Introduction}

Heart failure is a widespread disease in the world and is estimated to affect 5 million people in the United States alone

S. Nodari $(\bowtie) \cdot$ M. Triggiani · A. Manerba · G. Milesi · L. Dei Cas

Section of Cardiovascular Diseases, Department of Experimental and Applied Medicine, University of Brescia, Brescia, Italy e-mail: savinanodari@gmail.com
$[1,2]$. Annual costs related to the treatment of $\mathrm{HF}$ in the United States are estimated at $\$ 38$ billion, accounting for $5.4 \%$ of the healthcare budget [3]. The gold standard for treatment of HF has been based on medical guidelines published by groups such as the American Heart Association (AHA), American College of Cardiology (ACC), Heart Failure Society of America (HFSA), and the European Society of Cardiology (ESC) [4-6]. The recommended pharmacological therapy includes the use of angiotensin converting enzyme (ACE)-inhibitors, angiotensin receptor blockers (ARBs), diuretics, beta-blockers, and aldosterone antagonists. These pharmacological regimens have shown beneficial outcomes in Quality Of Life, morbidity, and/or mortality in HF patients [4]. Despite improvements in mortality, HF hospitalizations continue to rise, as an aging population lives longer secondary to increased overall life expectancy and improvements in HF therapy [7]. As more patients survive after acute myocardial infarction, thanks to improved revascularization strategies and better prevention of sudden cardiac death, they are often left with decreased LV function and HF [8]. Development of HF continues to be one of the strongest predictors of short-term readmission and mortality, with both rates remaining high despite current therapies [9]. Thus, while new medication options have improved morbidity, there remains substantial work to be done to reverse the disabling signs and symptoms of HF [10].

Over the past 30 years much epidemiological evidence and many experimental and clinical studies have supported a protective role of n-3 PUFAs in the prevention and treatment of cardiovascular diseases, particularly in coronary artery disease (CAD) and sudden cardiac death (SCD). Based on the results obtained from the GISSIPrevenzione [11], the European Society of Cardiology and the American Heart Association (AHA) have approved n-3 PUFAs supplementation (combination of Eicosapentaenoic 
Acid, EPA, and Docosahexaenoic Acid, DHA) at a dose of $1 \mathrm{~g} /$ day in patients with documented CAD, also suggesting a higher dietary intake of omega-3 (at least 2 meals of fish per week) for primary prevention of cardiovascular disease in patients without documented CAD $[12,13]$. PUFAs have also recently garnered increased attention in HF prevention and treatment. The purpose of this review is to summarize the evidence emerged from studies conducted so far on the effect of n-3 PUFAs in HF patients.

\section{Omega-3 Polyunsaturated fatty acids: metabolism and biological effects in heart failure}

Biochemical and biological aspects

Omega-3 are defined as essential fatty acids since they cannot be synthesized in our body, but a dietary intake, especially through oily fish (herring, mackerel, salmon, albacore tuna, sardines) or fish oil supplementation is needed [14]. There are two classes of PUFAs: omega 3 ( $\alpha$ - linolenic acid, LNA) and omega 6 (linoleic acid, LA). Both PUFAs are metabolized to long-chain fatty acids of 20 and 22 carbon atoms: LA is metabolized to arachidonic acid (AA) and LNA to EPA and DHA. They are important components of membrane's phospholipids and at this level they compete for the same enzymatic system, since their metabolism is completely separated and their interconversion is not possible [15]. When humans ingest fish or fish oil, EPA and DHA partially replace omega 6 fatty acids, especially arachidonic acid (AA), in all cells' membrane, favorably modifying omega6/omega 3 ratio. Thus a greater ingestion of EPA and DHA from fish or fish oil leads to: (1) a decreased production of thromboxane $\mathrm{A} 2$ leading to vasodilatation and reduction in platelet aggregation; (2) a decrease in leukotriene B4 synthesis, a powerful inducer of leukocyte chemotaxis and inflammation; (3) a decrease in cytokines levels (IL1, IL6, TNF $\alpha$ ) with a reduced inflammatory response to endothelial and tissue damage; (4) a modulation of ionic membrane channels conduction; (5) a modulation of intracellular signal transduction and gene expression through the regulation of several transcriptional nuclear factors. [16, 17].

A large number of structural and functional abnormalities may play an important role in the pathogenesis of HF: atherothrombosis, arrhythmias, abnormalities in energy metabolism, altered expression or function of contractile proteins, abnormalities of excitation-contraction coupling, cytoskeletal abnormalities, alterations in the beta-adrenergic-receptor signal transduction, ventricular hypertrophy and remodeling, and neurohormonal-cytokine changes [18]. The clinical benefit of n-3 PUFAs may be mediated by their effects on many of these processes and mechanisms (Table 1).
Table 1 Beneficial effects of n-3 PUFAs in heart failure

\begin{tabular}{ll}
\hline Hemodynamics & $\begin{array}{l}\text { Improve endothelium-dependent and } \\
\text { independent vasodilatation }\end{array}$ \\
& $\downarrow$ ET-1 \\
& $\uparrow$ NO \\
Antinflammatory & $\downarrow$ NF- $\kappa$ B activation \\
& Compete with AA for COX and 5- lipoxigenase \\
& enzymatic sites \\
Cardiac & $\uparrow$ ATP generation \\
energetics & $\downarrow$ O2 consumption \\
& $\downarrow$ sarcoplasmatic reticulum $\mathrm{Ca}^{2+}$ \\
Remodeling and & $\uparrow$ PPAR $\gamma \rightarrow \uparrow$ adiponectin \\
fibrosis & \\
Vascular & $\downarrow$ Platelet aggregation via $\downarrow$ TXA2 \\
& $\downarrow$ VCAM- 1, ELAM 1 , ICAM 1 \\
& $\downarrow$ Monocyte endothelial adherence via $\downarrow$ PAF \\
Antiarrhythmic & $\uparrow$ EPA: AA ratio in plasma membrane \\
& $\uparrow$ Ca ${ }^{2+}-\mathrm{Mg}^{2+}$ ATPase activity \\
& Inhibit fast voltage-dependent Na ${ }^{+}$channels \\
& Inhibit L-type Ca ${ }^{2+}$-channel
\end{tabular}

Metabolic effects: energy metabolism and mitochondrial function

Experimental data provided strong evidence that $\mathrm{n}-3 \mathrm{PU}-$ FAs are endogenous ligands for peroxisome proliferators activated receptors alpha and gamma (PPAR $\alpha$ and PPAR $\gamma$ ) $[19,20]$. LV hypertrophy and enlargement induced by pressure overload are associated with a decrease in the activity of mitochondrial enzymes involved in the fatty acid oxidation and energy transduction. Omega-3 PUFAs supplementation could preserve cardiac mitochondrial function by stimulating expression of proteins involved in cardiac lipid metabolism and mitochondrial function [21, 22]. Pepe and McLennan [23] showed that isolated perfused hearts from rats fed with fish oil, in comparison with control group, had reduced myocardial oxygen consumption without a decrease in LV power generation, at low or high workload, resulting in greater LV mechanic efficiency. The mechanism for this effect is not clear, but could be due to improved mitochondrial coupling and/or a decrease in ATP hydrolysis by processes not directly related to force generation.

Anti-inflammatory effects

Persistent inflammation, involving increased levels of inflammatory cytokines and vasoconstrictor eicosanoids, seems to play a pathogenic role in chronic HF by influencing heart contractility, inducing hypertrophy and 
promoting apoptosis, contributing to myocardial remodeling and to deterioration of renal function [24, 25].

The anti-inflammatory effect of n-3 PUFAs may be linked to their ability to modulate the nuclear transcription factor $(\mathrm{NF}-\kappa \mathrm{B})$ which is activated in $\mathrm{HF}$ [26-28]. The PPARs activation may lead to a suppression of NF- $\kappa \mathrm{B}$ and $\mathrm{NF}-\kappa \mathrm{B}$-regulated pro-inflammatory cytokines. In a small pilot study, conducted on 14 patients with HF (NYHA class III-IV) randomized to $8 \mathrm{~g} /$ day of EPA and DHA, there was a reduction in cytokine (TNF- $\alpha$ and IL-1) levels and an improvement in the percentage of body fat, suggesting that omega-3 can decrease inflammation and prevent cachexia in advanced HF patients [29]. In a recent study the treatment with n-3 PUFAs in elderly patient with HF significantly decreased plasma levels of TNF $\alpha$, IL-6, intracellular adhesion molecule 1 and NT-proBNP [30]. In another study, Eschen et al. [31] showed that n-3 PUFAs in 138 congestive $\mathrm{HF}$ patients did not significantly affect the plasma levels of circulating soluble cellular adhesion molecules and high sensitivity C-reactive protein, but they reduced $\mathrm{P}$-selectin levels confirming their effect on platelet and endothelial activation.

In adipocytes, n-3 PUFAs from fish oil increase expression, secretion and plasma levels of the antiinflammatory hormone adiponectin. Recent studies showed that adiponectin limits LV hypertrophy, remodeling, and contractile dysfunction in response to pressure overload and exerts anti-inflammatory effects [32]. The mechanisms for adiponectin-induced suppression of LV hypertrophy and dysfunction have been linked to activation of AMPactivated protein kinase (AMPK) [33, 34], but could also be due to inhibition of the serine-threonine kinase (Akt) [35]. However, despite the activation of AMPK by adiponectin, there is no direct clinical evidence of a strong relationship between adiponectin and $\mathrm{HF}$. In the Framingham Offspring Study, adiponectin was not associated with the development of new HF, but resistin, another adipokine, resulted in a link with HF [36]. Duda et al. [37, 38] in their studies in rats showed that EPA+DHA supplementation increased adiponectin and lowered TNF-alfa serum levels in a dose-dependent manner. Anyway omega-3 supplementation did not affect the activation of AMPK or Akt, suggesting that the protective effect of adiponectin could be mediated by other mechanisms, such as the modulation of NF- $\kappa \mathrm{B}$ activity.

\section{Hemodynamic effects}

Several studies have shown additional beneficial effects of n-3 PUFAs on HF development and progression by affecting hemodynamic and LV function [39]. These studies reported an improvement in endothelial function $[40,41]$ and a reduction in systemic vascular resistances by increasing the nitric oxide production [42, 43], a reduction in vasoconstrictive response to norepinephrine and angiotensin II, an improvement in arterial compliance and vascular response to vasodilators [44, 45]. These effects may justify the reduction of systolic $(-2.1 \mathrm{mmHg}, p<0.001)$ and diastolic $(-1.6 \mathrm{mmHg}, p<0.001)$ blood pressure (BP), reported in a recent meta-analysis of 36 randomized clinical trials, with an average dose of $3.7 \mathrm{~g} /$ day of $\mathrm{EPA}+\mathrm{DHA}$; this modest $\mathrm{BP}$ reduction resulted greater in older $(>45$ years) and hypertensive patients $(\mathrm{BP} \geq 140$ / $90 \mathrm{mmHg}$ ) [46].

Favorable effects of omega-3 administration on heart rate (HR) were also reported. A meta-analysis of 30 randomized studies showed that chronic administration of EPA+DHA (about $3.5 \mathrm{~g} /$ day, for a period longer than 12 weeks) can lead to a reduction in HR of about 2.5-3 beats/min in subjects with a baseline HR of 69 beats/min [47].

In addition, some experimental studies have shown that chronic administration of n-3 PUFAs can lead to an improvement in both systolic and diastolic myocardial function [48, 49] and can prevent the development of LV hypertrophy and systolic dysfunction induced by a pressure overload [38]. In a idiopathic dilated cardiomyopathy animal model, the long-term treatment with n-3 PUFAs induced a significant increase in isometric and isotonic contractile force of papillary muscles, an increase in the actin-myosin bridges [50], and a significant improvement in the peak rate of rise and fall of muscle tension, indicating a more efficient contraction and muscle relaxation, respectively [51]. These effects are probably related to the prevention of overloading of intracellular $\mathrm{Ca}^{2+}$ and reduction of this ion in the sarcoplasmic reticulum [52].

\section{Antiarrhythmic effects}

Tachyarrhythmias are one of the most important causes of death in patients with HF. Electrophysiological substrates of arrhythmias in $\mathrm{HF}$ are numerous and include LV remodeling, increased wall stress, heterogeneity of depolarization current, remodeling of ion channels and simpathovagal unbalance. Myocardial ischemia, electrolyte abnormalities, drugs, or fluctuations in the autonomic system may be the triggers [53]. Different mechanisms by which n-3 PUFAs may exert their antiarrhythmic effects have been reported, including anti-inflammatory and antithrombotic effects, modulation of ion channels $\left(\mathrm{Ca}^{2+}\right.$ and $\mathrm{Na}^{+}$) and simpathovagal balance [54-57].

The antiarrhythmic properties of n-3 PUFAs have been demonstrated in experimental models [58, 59] and in clinical trials in patients with CAD $[11,60]$ while the trials conducted in patients with an implantable cardioverter defibrillator (ICD) have yielded controversial results [61- 
64]. These conflicting data may be due to several methodological differences regarding study population and follow-up, but the use of different formulations and doses of n-3 PUFAs may represent an important explanation. In fact, although the SCD reduction in the GISSI-Prevenzione was obtained with low dose of omega-3, it was demonstrated that the relative risk of SCD is associated with baseline blood levels of n-3 PUFAs [65] and their antiarrhythmic effects in ischemic patients with ICD are related to the increased n-3 PUFAs' concentration in cell membranes during treatment [64].

In addition, little is yet known about the impact of different etiology of HF (ischemic and non-ischemic) on the results of treatment with omega-3. In our study [66], we evaluated the effectiveness of n-3 PUFAs in reducing the arrhythmic risk in patients with idiopathic dilated cardiomyopathy and with frequent or repetitive ventricular tachyarrhythmia episodes (NSVT), despite optimized drug therapy. At the end of the 6-month follow-up, we observed in n-3 PUFAs group, in comparison with placebo group, a significant reduction in the number and duration of episodes of NSVT $(p=0.0002)$ and a decrease of average HR of VT at Holter monitoring $(p=0.0003)$, with an improvement of heart rate variability (HRV) $(p<0.001)$ and a reduction of catecholamine and cytokine serum levels $(p<0.001)$. The $n-6 / n-3$ PUFA ratio was significantly reduced in the treatment group from 1.12 to 3.48 . As noted in other series $[67,68]$, the reduction in plasma levels of catecholamines and cytokines, in addition to increased HRV, were related to the increase in concentrations of EPA and DHA, confirming the impact of n-3 PUFAs on important pathogenic mechanisms of arrhythmias in nonischemic HF patients.

\section{n-3PUFAs in heart failure: epidemiological and interventional studies}

EPA and DHA modulate favorably several factors related to the pathophysiology of $\mathrm{HF}$, including the levels of fatty acids, energy metabolism of myocytes, mitochondrial function, inflammatory response and endothelial function. All these effects could contribute to prevent or delay the development and progression of HF. However, while there are more data in the literature to support a role of $\mathrm{EPA}+\mathrm{DHA}$ supplementation in primary and secondary prevention of CAD [69, 70], until now there are only few evidences to support their benefit in HF patients.

Mozaffarian et al. [71] investigated the association between fish consumption and incidence of $\mathrm{HF}$ in the Cardiovascular Health Study, a population-based cohort study including 4,738 adults, aged >65 years. Their hypothesis was that consumption of tuna and other broiled or baked fish, but not fried fish, would be associated with a lower incidence of HF. During 12-years follow-up, 955 participants developed HF. In multivariate-adjusted analyses, tuna/other fish consumption was inversely associated with incident $\mathrm{HF}$.

Another study [72] analyzed the association between fatty acid levels and HF incidence in 15,792 subjects aged between 45 and 64 years. During 14.3 years of follow-up 197 cases of HF were identified. After adjustment for age and other confounders, while the levels of saturated fatty acids (palmitic acid) were directly correlated with the incidence of HF in both sexes, levels of n-3 PUFAs showed an inverse correlation in women. Finally, Levitan et al. [73] have studied the possible correlation between HF incidence and fish consumption in a cohort of subjects aged 45-79 years in Sweden. In contrast to the study of Mozaffarian [71], the association between fish consumption and the incidence of $\mathrm{HF}$ showed U-shaped distribution in multivariate analysis of consumption quintiles: patients in the first quintile $(0.15 \mathrm{~g} /$ day of fish) had a lower percentage of HF although not statistically significant (-12\%) compared to no fish consumption, as well as patients in the third quintiles ( $0.36 \mathrm{~g} /$ day) had a reduced risk of $\mathrm{HF}$ by $33 \%$ compared to those in the fifth quintiles ( $0.76 \mathrm{~g} / \mathrm{day})$. The authors justify this result by assuming that subjects with a greater intake of fish could be more vulnerable to the adverse effects of fish's contaminants (mercury, dioxides) or could have more cardiovascular risk factors (hypercholesterolemia and hypertension).

The first clinical study on the administration of $n-3$ PUFAs in patients with HF was the GISSI-HF Investigators et al. [74]. A total of 6,975 HF patients (NYHA class II-IV) were randomized to $1 \mathrm{~g} /$ day of $\mathrm{n}-3$ PUFAs (850-882 $\mathrm{mg} /$ day $\mathrm{EPA}+\mathrm{DHA}$ ) as ethyl ester or to matching placebo and followed for a median of 3.9 years. Results showed a significant reduction in primary endpoints, total mortality $(-9 \%$, $P<0.05)$ and mortality and hospitalizations for $\mathrm{CV}$ disease $(-8 \%, P<0.01)$ in $\mathrm{n}-3$ PUFAs group in comparison with placebo, after adjustment for possible confounding factors (previous HF hospitalization, pacemaker, aortic stenosis). Although these results seem to be lower than expected, the improvement of clinical outcomes was obtained in addition to optimal medical therapy (including beta-blockers, ACE inhibitors, AT1 and aldosterone antagonists) and correspond to a NNT of 56 to prevent one death and NNT of 44 to prevent an event (death or cardiovascular hospitalization).

In addition, more recently, the results of the echocardiographic substudy of GISSI-HF evidenced that chronic administration of omega-3, in contrast to treatment with rosuvastatin, was associated with a significant increase of ejection fraction (EF) when compared to placebo (8.1 vs. $6.3 \%$ at 1 year, 11.1 vs. $8.2 \%$ at 2 years, 11.5 vs. $9.9 \%$ at 3 years, respectively; $p=0.005$ ) [75]. These results 
suggest that the improved survival observed in HF patients treated with omega-3 could be attributed to an improvement of LV systolic function.

Despite this evidence, no study had specifically examined the effects of omega- 3 on the ventricular function and functional capacity in HF patients. To verify these effects, we enrolled 133 patients with chronic HF due to nonischemic dilated cardiomyopathy, $\mathrm{EF}<45 \%$, stable clinical conditions on evidence-based medical treatment at maximum tolerated target doses for at least 6 months, into a double-blind, placebo-controlled, two-arm study [76]. Participants were randomly allocated to active treatment (1.0 g gelatin capsules containing $850-882 \mathrm{mg}$ of eicosapentaenoic (EPA) and docosahexaenoic (DHA) ethyl esters in the average ratio EPA/DHA of 0.9:1.5) or to placebo (1.0 g gelatin capsules containing olive oil). The treatment dose was five capsules daily for the first month followed by two capsules daily for the rest of the study. The primary outcome was the change in LV systolic function expressed as EF, while the secondary outcomes included: LV diastolic function assessed by echocardiography; functional capacity assessed by cardiopulmonary exercise testing (CPET); and NYHA functional class. At the time of enrollment and at 12-month follow-up patients underwent a physical examination, ECG, blood draw for complete blood count, comprehensive chemistry panel, inflammatory cytokines, including TNF- $\alpha$, IL- 6 and IL-1, and serum free fatty acids (FFAs) levels, echocardiographic study and CPET. We found that at 12 months after randomization, the n-3 PUFAs group and the placebo group differed significantly $(p<0.001)$ in regard to LV ejection fraction and the other echocardiographic variables and in parameters of functional capacity. In particular, the n-3 PUFA group showed, at 1 year of follow-up compared with baseline, a significant increase in EF $(10.4 \pm 9.5 \%, p<0.001)$ (Fig. $1)$, a significant reduction in diameter (EDD $-1.8 \%$, $p<0.0001$; ESD $-4.5 \%, p<0.001)$ and ventricular volume (EDV $-2.5 \%, p<0.001$; ESV $-7.5 \% p<0.0001$ ). In contrast, in the placebo group at the end of the study there was a significant reduction in EF as well as a statistically significant increase in ventricular diameters and volumes. In the group treated with n-3 PUFAs was also observed an improvement of diastolic function was also observed, expressed by a significant reduction of diastolic dysfunction score $(-4.74 \%, p=0.004)$ compared with an increase in this parameter in the placebo group $(10.1 \%$, $p<0.001$ ). Positive results of treatment with omega- 3 were observed for the functional capacity, expressed by an increase of peak VO2 $(6.2 \%, p<0.001)$ and duration of exercise $(7.7 \%, p<0.001)$ to cardiopulmonary stress test and a reduction in NYHA functional class (from $1.88 \pm 0.33$ to $1.61 \pm 0.49, p<0.001)$. In the placebo group was shown at 12-month follow-up to a greater
Ejection Fraction

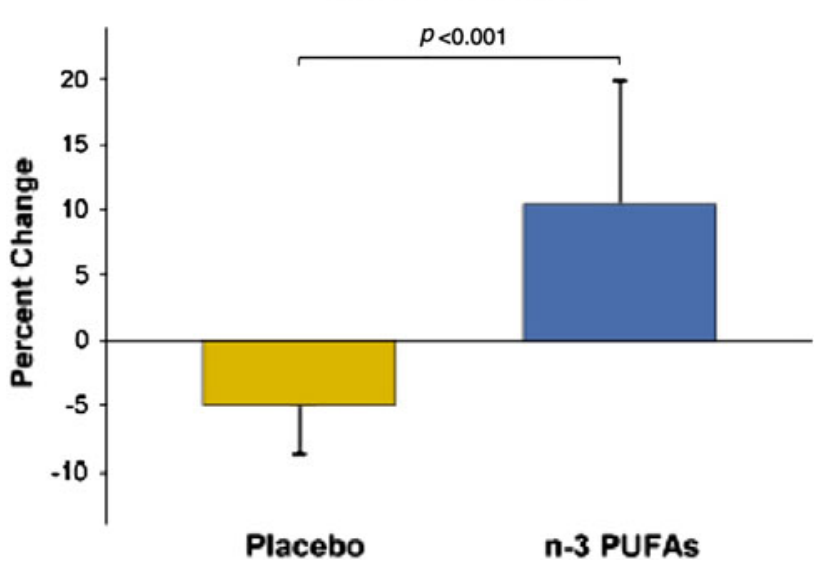

Fig. 1 Variations of the EF in the two study groups (from Nodari [76])

degree of functional impairment was shown at a decline in oxygen consumption and an increase in NYHA class. During follow-up, the serum levels of inflammatory cytokines (TNF-alpha, IL-6, IL-1) increased significantly in the placebo group, while decreased in omega-3 group $(p<0.001)$. Of interest, throughout follow-up, lower hospitalization rates for cardiovascular causes (15 vs. 39\%, respectively; $p=0.002$ ) and for HF (6 vs. 30\%, respectively; $p=0.0002)$ were noted in the n-3 PUFAs-treated patients compared to those on placebo $(p<0.0002)$. The results of our study suggest that n-3 PUFAs may act favorably on ventricular function, both systolic and diastolic, on left ventricular remodeling and functional capacity, resulting in a lower incidence of HF and cardiovascular hospitalizations.

\section{Dose and optimal ratio of DHA/EPA}

The potential favorable mechanisms of $n-3$ PUFAs in CV diseases seem closely related to increased concentrations of EPA and DHA in cell membranes [77]. The antiplatelet, anti-inflammatory and lipid-lowering effects occur with relatively high doses of EPA and DHA (3-4 g/day); on the other hand the antiarrhythmic effect, the prevention of SCD and the improvement in HF can be achieved with lower doses (500-1,000 mg/day). The optimal dose and the ratio of DHA and EPA has not been defined yet. DHA and EPA are found in most kinds of fishes, particularly in fatty fishes, usually in a 2:1 ratio, while pharmacological preparations typically have a ratio of 2:3 or less [78]. Moreover, while the administration of DHA can also increase levels of EPA, the reverse process from EPA to DHA in humans does not occur [79]. DHA is more represented than EPA in myocyte membrane and its administration (alone or in 
combination with EPA) seems to be more effective than only EPA in preventing arrhythmias and SCD. Although the beneficial effects on arrhythmias occurred at low doses, the relative risk of SCD was inversely correlated with baseline blood levels of n-3 PUFAs and protective effect in patients at high risk of fatal arrhythmias, such as ICD patients, was correlated with the concentration achieved during treatment [64]. Similarly, an inverse correlation was confirmed between tissue levels of DHA and CAD [80]. A recent study [81] conducted in patients with HF showed an association between DHA concentrations and degree of LV remodeling: for diastolic diameter between 68 and $90 \mathrm{~mm}$ (upper tertile) compared with diameters between 48 and $61 \mathrm{~mm}$ (lower tertile), as well as EF values between 9 and $25 \%$ (lower tertile) compared with values of EF from 35 to $50 \%$ (upper tertile), serum concentrations of DHA were significantly lower ( 1 vs. $1.3 \%, p<0.001)$.

In our studies [77] we observed a significant inverse association between baseline levels of omega-3 (particularly DHA) and magnitude of LV remodeling and dysfunction, and between increased concentrations of DHA and the improvement in these parameters at the end of follow-up. These results suggest a prominent role of DHA in the pathophysiology and then in the treatment of ventricular dysfunction and remodeling.

As part of a therapy "tailored" for each patient, DHA could be a new sensitive biomarker for monitoring and diagnosis of systolic dysfunction and dilation in patients with LV systolic HF, or could be used to determine the optimal dosage and to monitor the response to therapy in each patient.

\section{Conclusion and future perspectives}

In recent years there has been increasing evidence from observational, experimental and clinical studies about the beneficial effects of omega- 3 in the prevention and treatment of cardiovascular disease and their possible mechanisms of action. However, sometimes conflicting results have raised doubts and uncertainties. This is partly motivated by the insufficient knowledge about the role of omega-3 in complex biological mechanisms and cellular system and by the publication of studies which seem similar, but have significant differences in methodology (case studies, dose, duration of follow-up, etc.) which can lead to different results.

The GISSI-HF has not only provided important evidence concerning the improvement of prognosis in patients with HF treated with omega-3, but it also raised many questions about the mechanisms underlying the favorable effect of $n-3$ PUFA in HF, in which patient could have more benefit from the treatment with n-3 PUFA and which is the optimal dose.
In our experience, the n-3 PUFA supplementation in addition to an optimized medical therapy, was associated not only with a reduction of arrhythmic risk, but also to an improvement in $\mathrm{EF}$ and functional capacity, important prognostic factors in HF patients.

Targeted studies are needed to respond to specific questions, first the optimal dose to use and the optimal ratio of EPA and DHA. The history of n-3 PUFAs in HF is definitely still developing, but it is not too early to start listening to its lessons. Among these, for example, there is the importance of undertaking studies on the effects of metabolic therapy in patients with advanced-stage of HF, in which therapeutic intervention may help to recover dysfunctional, but vital, myocardium.

Conflict of interest None.

\section{References}

1. McCullough PA, Philbin EF, Spertus JA, Kaatz S et al (2002) Confirmation of a heart failure epidemic: findings from the Resource Utilization Among Congestive Heart Failure (REACH) study. J Am Coll Cardiol 39(1):60-69

2. Rosamond W, Flegal K, Furie K, Go A et al (2008) Heart disease and stroke statistics-2008 update: a report from the American Heart Association Statistics Committee and Stroke Statistics Subcommittee. Circulation 117(4):e25-e146

3. O'Connell JB (2000) The economic burden of heart failure. Clin Cardiol 23(3 Suppl):III6-10

4. Hunt SA, Abraham WT, Chin MH, Feldman AM et al (2005) ACC/AHA 2005 Guideline Update for the Diagnosis and Management of Chronic Heart Failure in the Adult: a report of the American College of Cardiology/American Heart Association Task Force on Practice Guidelines (Writing Committee to Update the 2001 Guidelines for the Evaluation and Management of Heart Failure): developed in collaboration with the American College of Chest Physicians and the International Society for Heart and Lung Transplantation: endorsed by the Heart Rhythm Society. Circulation 112(12):e154-e235

5. Heart Failure Society Of America (2006) HFSA 2006 Comprehensive Heart Failure Practice Guideline. J Card Fail 12(1):e1-e2

6. Dickstein K, Cohen-Solal A, Filippatos G, McMurray JJ, et al (2008) ESC Guidelines for the diagnosis and treatment of acute and chronic heart failure 2008: The Task Force for the Diagnosis and Treatment of Acute and Chronic Heart Failure 2008 of the European Society of Cardiology. Developed in collaboration with the Heart Failure Association of the ESC (HFA) and endorsed by the European Society of Intensive Care Medicine (ESICM). Eur Heart J 29(19):2388-2442

7. Fang J, Mensah GA, Croft JB, Keenan NL (2008) Heart failurerelated hospitalization in the U.S., 1979 to 2004. J Am Coll Cardiol 52(6):428-434

8. Tang WH, Francis GS (2003) Trends and treatment of heart failure developing after acute myocardial infarction. Am Heart Hosp J 1(3):216-218

9. Wu AH, Parsons L, Every NR, Bates ER (2002) Hospital outcomes in patients presenting with congestive heart failure complicating acute myocardial infarction: a report from the Second National Registry of Myocardial Infarction (NRMI-2). J Am Coll Cardiol 40(8):1389-1394 
10. Butler J, Kalogeropoulos A (2008) Worsening heart failure hospitalization epidemic we do not know how to prevent and we do not know how to treat!. J Am Coll Cardiol 52(6):435-437

11. Gissi Prevenzione Investigators (1999) Dietary supplementation with $n-3$ polyunsaturated fatty acids and vitamin $\mathrm{E}$ after myocardial infarction: results of the GISSI-Prevenzione trial. Gruppo Italiano per lo Studio della Sopravvivenza nell'Infarto miocardico. Lancet 354:447-455

12. Van de Werf F, Ardissino D, Betriu A, Cokkinos DV et al (2003) Management of acute myocardial infarction in patients presenting with ST-segment elevation. The Task Force on the Management of Acute Myocardial Infarction of the European Society of Cardiology. Eur Heart J 24:28-66

13. Kris-Etherton PM, Harris WS, Appel LJ (2002) for the American Heart Association Nutrition Committee. Fish consumption, fish oil, omega-3 fatty acids, and cardiovascular disease. Circulation 106:2747-2757

14. Rogans JA (1987) More on fish oil. N Engl J Med 316:624-628

15. Dei Cas L, Nodari S (2003) Ruolo degli acidi grassi poliinsaturi PUFA n-3 nella prevenzione della morte improvvisa. Excerpta Medica, pp 1-80

16. Weber PC (1986) Fischer 5, von Schacky C, Lorenz R, et al. Dietary omega-3 polyunsaturated fatty acids and eicosanoid formation in man. In: Simopoulos AP, Kifer RR, Martin RE (eds) Health effects of polyunsaturated fatty acids in seafoods. Academic Press, Orlando, FL, pp 49-60

17. Lewis RA, Lee TH, Austen KF (1986) Effects of omega-3 fatty acids on the generation of products of the 5-lipoxygenase pathway. In: Simopoulos AP, Kifer RR, Martin RE (eds) Health effects of polyunsaturated fatty acids in seafoods. Academic Press, Orlando, FL, pp 227-238

18. Braunwald E, Bristow (2000) Congestive heart failure: fifty years of progress. Circulation 102(20 Suppl 4):IV14-23

19. Xu HE, Lambert MH, Montana VG, Parks DJ et al (1999) Molecular recognition of fatty acids by peroxisome proliferatoractivated receptors. Mol Cell 3:397-403

20. Kliewer SA, Sundseth SS, Jones SA, Brown PJ et al (1997) Fatty acids and eicosanoids regulate gene expression through direct interactions with peroxisome proliferator-activated receptors alpha and gamma. Proc Natl Acad Sci U S A 94(9):4318-4323

21. Sack MN, Rader TA, Park S, Bastin J et al (1996) Fatty acid oxidation enzyme gene expression is downregulated in the failing heart. Circulation 94:2837-2842

22. Pepe S, McLennan PL (2007) (n-3) Long chain PUFA dosedependently increase oxygen utilization efficiency and inhibit arrhythmias after saturated fat feeding in rats. J Nutr 137(11): 2377-2383

23. Pepe S, McLennan PL (2002) Cardiac membrane fatty acid composition modulates myocardial oxygen consumption and postischemic recovery of contractile function. Circulation 105: 2303-2308

24. Castellani S, Paladini B, Paniccia R, Di Serio C et al (1997) Increased renal formation of thromboxane A2 and prostaglandin F2 alpha in heart failure. Am Heart J 133(1):94-100

25. Aukrust P, Gullestad L, Ueland T, Damås JK et al (2005) Inflammatory and anti-inflammatory cytokines in chronic heart failure: potential therapeutic implications. Ann Med 37(2):74-85

26. Valen G, Yan ZQ, Hansson GK (2001) Nuclear factor kappa-B and the heart. J Am Coll Cardiol 38:307-314

27. Wong SC, Fukuchi M, Melnyk P, Rodger I et al (1998) Induction of cyclooxygenase- 2 and activation of nuclear factor-kappaB in myocardium of patients with congestive heart failure. Circulation 98:100-103

28. Matsumori A, Sasayama S (2001) The role of inflammatory mediators in the failing heart: immunomodulation of cytokines in experimental models of heart failure. Heart Fail Rev 6:129-136
29. Mehra MR, Lavie CJ, Ventura HO, Milani RV (2006) Fish oils produce anti-inflammatory effects and improve body weight in severe heart failure. J Heart Lung Transplant 25(7):834-838

30. Zhao YT, Shao L, Teng LL, Hu B et al (2009) Effects of n-3 polyunsaturated fatty acid therapy on plasma inflammatory markers and $\mathrm{N}$-terminal pro-brain natriuretic peptide in elderly patients with chronic heart failure. J Int Med Res 37(6):18311841

31. Eschen O, Christensen JH LA, Rovere MT, Romano $\mathrm{P}$ et al (2010) Effects of marine n-3 fatty acids on circulating levels of soluble adhesion molecules in patients with chronic heart failure. Cell Mol Biol. 56(1):45-51 Noisy-le-grand

32. Shibata R, Ouchi N, Ito M, Kihara S et al (2004) Adiponectinmediated modulation of hypertrophic signals in the heart. Nat Med 10:1384-1389

33. Shibata R, Ouchi N, Ito M, Kihara S et al (2004) Adiponectinmediated modulation of hypertrophic signals in the heart. Nat Med 10:1384-1389

34. Hopkins TA, Ouchi N, Shibata R, Walsh K (2007) Adiponectin actions in the cardiovascular system. Cardiovasc Res 74:11-18

35. Wang Y, Lam JB, Lam KS, Liu J et al (2006) Adiponectin modulates the glycogen synthase kinase-3beta/beta-catenin signaling pathway and attenuates mammary tumorigenesis of MDAMB-231 cells in nude mice. Cancer Res 66:11462-11470

36. Frankel DS, Vasan RS, D'Agostino RB Sr et al (2009) Resistin, adiponectin, and risk of heart failure the Framingham offspring study. J Am Coll Cardiol 53:754-762

37. Duda MK, O'Shea KM, Lei B et al (2007) Dietary supplementation with omega-3 PUFA increases adiponectin and attenuates ventricular remodelling and dysfunction with pressure overload. Cardiovasc Res 76:303-310

38. Duda MK, O'Shea KM, Tintinu A, Xu W et al (2009) Fish oil, but not flaxseed oil, decreases inflammation and prevents pressure overload-induced cardiac dysfunction. Cardiovasc Res 81(2):319-327

39. Mozaffarian D, Gottdiener JS, Siscovick DS (2006) Intake of tuna or other broiled or baked fish versus fried fish and cardiac structure, function, and hemodynamics. Am J Cardiol 97(2):216-222

40. Matsumoto T, Nakayama N, Ishida K et al (2009) Eicosapentaenoic acid improves imbalance between vasodilator and vasoconstrictor actions of endothelium-derived factors in mesenteric arteries from rats at chronic stage of type 2 diabetes. J Pharmacol Exp Ther 329:324-334

41. Morgan DR, Dixon LJ, Hanratty CG, El-Sherbeeny N et al (2006) Effects of dietary omega-3 fatty acid supplementation on endothelium-dependent vasodilation in patients with chronic heart failure. Am J Cardiol 97(4):547-551

42. Omura M, Kobayashi S, Mizukami Y et al (2001) Eicosapentaenoic acid (EPA) induces $\mathrm{Ca}(2+)$-independent activation and translocation of endothelial nitric oxide synthase and endothelium-dependent asorelaxation. FEBS Lett 487:361-366

43. Harris WS, Rambjør GS, Windsor SL, Diederich D (1997) n-3 fatty acids and urinary excretion of nitric oxide metabolites in humans. Am J Clin Nutr 65(2):459-464

44. Mori TA, Watts GF, Burke V et al (2000) Differential effects of eicosapentaenoic acid and docosahexaenoic acid on vascular reactivity of the forearm microcirculation in hyperlipidemic, overweight men. Circulation 102:1264-1269

45. Mcveigh GE, Brennan GM, Cohn JN et al (1994) Fish oil improves arterial compliance in non-insulin-dependent diabetes mellitus. Arterioscler Thromb 14:1425-1429

46. Geleijnse JM, Giltay EJ, Grobbee DE, Donders AR et al (2002) Blood pressure response to fish oil supplementation: metaregression analysis of randomized trials. J Hypertens 20(8):14931499 
47. Mozaffarian D, Geelen A, Brouwer IA et al (2005) Effect of fish oil on heart rate in humans: a meta-analysis of randomized controlled trials. Circulation 112:1945-1952

48. Charnock JS, McLennan PL, Abeywardena MY (1992) Dietary modulation of lipid metabolism and mechanical performance of the heart. Mol Cell Biochem 116:19-25

49. McLennan PL, Barnden LR, Bridle TM, Abeywardena MY et al (1992) Dietary fat modulation of left ventricular ejection fraction in the marmoset due to enhanced filling. Cardiovasc Res 26:871-877

50. Vargiu R, Littarru GP, Fraschini M, Perinu A et al (2010) Enhancement of shortening velocity, power, and acto-myosin crossbridge (CB) kinetics following long-term treatment with propionyl-L-carnitine, coenzyme Q10, and omega-3 fatty acids in BIO TO-2 cardiomyopathic Syrian hamsters papillary muscle. Biofactors. 36(3):229-239

51. Vargiu R, Littarru GP, Faa G, Mancinelli R (2008) Positive inotropic effect of coenzyme Q10, omega-3 fatty acids and propionyl-L-carnitine on papillary muscle force-frequency responses of BIO TO-2 cardiomyopathic Syrian hamsters. Biofactors 32(1-4):135-144

52. Berecki G, Den Ruijter HM, Verkerk AO, Schumacher CA et al (2007) Dietary fish oil reduces the incidence of triggered arrhythmias in pig ventricular myocytes. Heart Rhythm 4(11):1452-1460

53. Engelstein ED (2003) Prevention and management of chronic heart failure with electrical therapy. Am J Cardiol 91(9A):62F$73 \mathrm{~F}$

54. Kang JX, Leaf A (1996) Evidence that free polyunsaturated fatty acids modify $\mathrm{Na}+$ channels by directly binding to the channel proteins. Proc Natl Acad Sci USA 93:3542-3546

55. Leaf A, Kang JX, Xiao YF, Billman GE (2003) Clinical prevention of sudden cardiac death by $n-3$ polyunsaturated fatty acids and mechanism of prevention of arrhythmias by n-3 fish oils. Circulation 107:2646-2652

56. Seidler T, Hasenfuss G, Maier LS (2007) Targeting altered calcium physiology in the heart: translational approaches to excitation, contraction, and transcription. Physiology (Bethesda) 22:328-334

57. Mozaffarian D, Geelen A, Brouwer IA et al (2005) Effect of fish oil on heart rate in humans: a meta-analysis of randomized controlled trials. Circulation 112:1945-1952

58. Billman GE, Hallaci H, Leaf A (1994) Prevention of ischemiainduced ventricular fibrillation by omega 3 fatty acids. Proc Natl Acad Sci USA 91:4427-4430

59. Billman GE, Kang JX, Leaf A (1999) Prevention of sudden cardiac death by dietary pure omega- 3 polyunsaturated fatty acids in dogs. Circulation 99:2452-2457

60. Bucher HC, Hengstler P, Sdiindler C, Meier G (2002) N-3 polyunsaturated fatty acids in coronary heart disease: a metaanalysis of randomized controlled trials. Am J Med 112:298-308

61. Raitt MH, Connor WE, Morris C et al (2005) Fish oil supplementation and risk of ventricular tachycardia and ventricular fibrillation in patients with implantable defibrillators: a randomized controlled trial. JAMA 293:2884-2891

62. Leaf A, Albert CM, Josephson M et al (2005) Prevention of fatal arrhythmias in high-risk subjects by fish oil n-3 fatty acid intake. Circulation 112:2762-2768

63. Brouwer IA, Zock PL, Camm AJ et al (2006) Effect of fish oil on ventricular tachyarrhythmia and death in patients with implantable cardioverter defibrillators: the Study on Omega-3 Fatty Acids and Ventricular Arrhythmia (SOFA) randomized trial. JAMA 295:2613-2619
64. Burr ML, Fehily AM, Gilbert JF, Rogers S et al (1989) Effects of changes in fat, fish, and fibre intakes on death and myocardial reinfarction: diet and reinfarction trial (DART). Lancet 2(8666):757-761

65. Albert CM, Campos H, Stampfer MJ, Ridker PM et al (2002) Blood levels of long-chain n-3 fatty acids and the risk of sudden death. N Engl J Med 346(15):1113-1118

66. Nodari S, Metra M, Milesi G, Manerba A et al (2009) The Role of n-3 PUFAs in Preventing the Arrhythmic Risk in Patients with Idiopathic Dilated Cardiomyopathy. Cardiovasc Drugs Ther 23:5-15

67. Christensen JH, Gustenhoff P, Korup E et al (1996) Effect of fish oil on heart rate variability in survivors of myocardial infarction: a double blind randomised controlled trial. BMJ 312:677-678

68. Christensen JH, Korup E, Aaroe J et al (1997) Fish consumption, n-3 fatty acids in cell membranes, and heart rate variability in survivors of myocardial infarction with left ventricular dysfunction. Am J Cardiol 79:1670-1673

69. Mozaffarian D, Rimm EB (2006) Fish intake, contaminants, and human health: evaluating the risks and the benefits. JAMA. 296(15):1885-1899

70. Mozaffarian D (2008) Fish and n-3 fatty acids for the prevention of fatal coronary heart disease and sudden cardiac death. Am J Clin Nutr 87(6):1991S-1996S

71. Mozaffarian D, Bryson CL, Lemaitre RN, Burke GL, Siscovick DS (2005) Fish intake and risk of incident heart failure. J Am Coll Cardiol 45:2015-2021

72. Yamagishi K, Nettleton JA, Folsom AR (2008) Plasma fatty acid composition and incident heart failure in middle-aged adults: the Atherosclerosis Risk In Communities (ARIC) study. Am Heart J 156:965-974

73. Levitan EB, Wolk A, Mittleman M (2009) Fish consumption, marine omega-3 fatty acids and incidence of heart failure: a population-based prospective study of middle-aged and elderly men. Eur Heart J 30:1495-1500

74. GISSI-HF Investigators et al (2008) Effect of n-3 polyunsaturated fatty acids in patients with chronic heart failure (the GISSI-HF trial): a randomised, double-blind, placebo-controlled trial. Lancet 372(9645):1223-1230

75. Ghio S, Scelsi L, Latini R, Masson S et al (2010) Effects of n-3 polyunsaturated fatty acids and of rosuvastatin on left ventricular function in chronic heart failure: a substudy of GISSI-HF trial. Eur J Heart Fail 12(12):1345-1353

76. Nodari S, Triggiani M, Campia U, Manerba A et al (2011) Effects of $n-3$ polyunsaturated fatty acids on left ventricular function and functional capacity in patients with dilated cardiomyopathy. J Am Coll Cardiol 57(7):870-879

77. Harris WS (2007) Omega-3 fatty acids and cardiovascular disease a case for omega-3 index as a new risk factor. Pharmacol Res 55:217-223

78. USDA Agricultural Research Service. Nutrient Data Laboratory. Available at: http://www.ars.usda.gov/nutrientdata. Accessed January 23, 2008

79. Park Y, Harris WS (2003) Omega-3 fatty acid supplementation accelerates chylomicron triglyceride clearance. J Lipid Res 44(3):455-463 Epub 2002 Dec 1

80. Harris WS, Poston WC, Haddock CK (2007) Tissue n-3 and n-6 fatty acids and risk for coronary heart disease events. Atherosclerosis. 193(1): 1-10

81. Rupp H, Rupp TP, Alter P, Maisch B (2010) Inverse shift in serum polyunsaturated and monounsaturated fatty acids is associated with adverse dilatation of the heart. Heart 96(8):595-598 